Provided for non-commercial research and education use. Not for reproduction, distribution or commercial use.

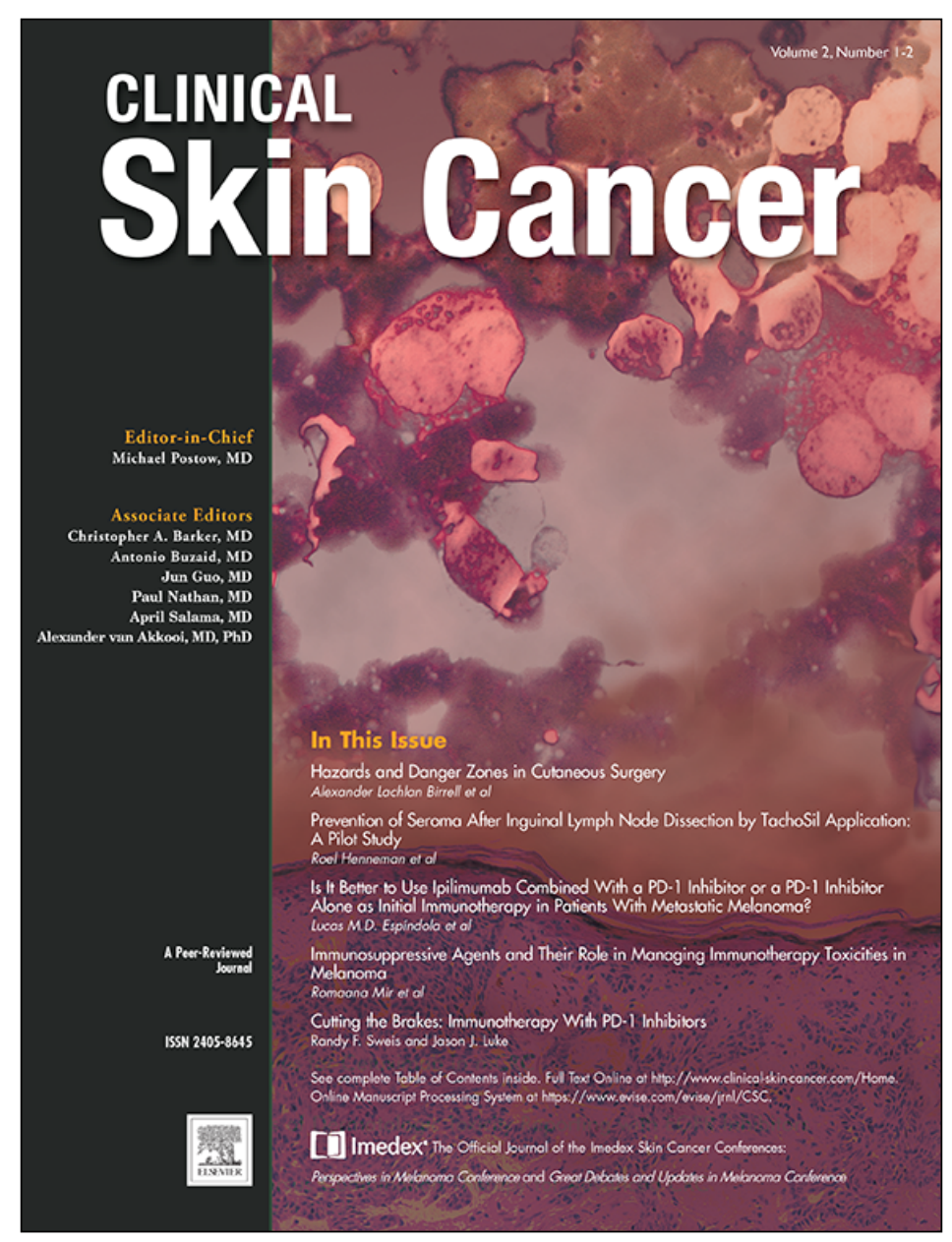

This article appeared in a journal published by Elsevier. The attached copy is furnished to the author for internal non-commercial research and education use, including for instruction at the author's institution and sharing with colleagues.

Other uses, including reproduction and distribution, or selling or licensing copies, or posting to personal, institutional or third party websites are prohibited.

In most cases authors are permitted to post their version of the article (e.g. in Word or Tex form) to their personal website or institutional repository. Authors requiring further information regarding Elsevier's archiving and manuscript policies are encouraged to visit:

http://www.elsevier.com/authorsrights 


\title{
Leishmaniasis and Malignancy: A Review and Perspective
}

\author{
Mohamed Ahmed Naif Al-Kamel
}

\begin{abstract}
Leishmaniasis, a widely prevalent disease throughout tropical and subtropical regions of the world, is a chronic protozoan infection of humans and mammals that remains grossly underreported and causes significant global morbidity and mortality. Different species of the intracellular protozoan parasites of the genus Leishmania can cause human leishmaniasis, resulting in various visceral, cutaneous, and mucocutaneous manifestations. The risk of leishmaniasis manifesting as a more severe entity is dependent on the infecting parasites' immune evasion potential. Some causal associations between leishmaniasis and malignancy have been evidenced in experimental animals and humans. Leishmania spp. infection can play a significant, direct or indirect, role in the pathogenesis and prognosis of some malignant disorders through numerous pathophysiologic cascades. Aging, chronic ultraviolet radiation exposure, and popular treatment abuse (eg, chameleon saliva, cactus recipes, corrosive chemicals, and topical steroids) have been proposed as the principal triggering factors. Malignancy should be considered in the differential diagnosis of leishmaniasis in endemic regions, such as Yemen. Understanding this relationship could enrich the provision of early diagnosis, proper management, and prompt control of cancer.
\end{abstract}

Clinical Skin Cancer, Vol. 2, No. 1-2, 54-8 @ 2017 Elsevier Inc. All rights reserved.

Keywords: Association, Cancer, Carcinogenesis, Dermatology, RLCC

\section{Introduction}

Leishmaniasis, a chronic parasitic infection of humans and mammals, is a neglected disease that is widely prevalent in many tropical and subtropical regions of the world. It remains grossly underreported and predominately affects children and women, with serious clinical consequences. Different species of the intracellular protozoan parasites of the genus Leishmania can cause human leishmaniasis, resulting in various visceral, cutaneous, and mucocutaneous manifestations. These parasites are transmitted to a human reservoir or host through bites of a blood-sucking vector, female sandflies. Visceral leishmaniasis (VL), which is lethal if left untreated, and cutaneous leishmaniasis (CL), which can affect the skin and/or mucous membranes with a broad spectrum of clinical manifestations, including dissemination (disseminated CL), are the most common clinical entities. ${ }^{1-3}$

The risk of leishmaniasis manifesting as a more severe entity is dependent on the infecting parasites' immune evasion potential, which is controlled by numerous factors, such as the infecting

Regional Leishmaniasis Control Center, and Department of Dermatology, Sana'a University Hospital, Sana'a, Yemen

Submitted: Oct 2, 2017; Revised: Oct 25, 2017; Accepted: Oct 30, 2017; Epub: Nov 6, 2017

Address for correspondence: Mohamed Ahmed Naif Al-Kamel, MD, Regional Leishmaniasis Control Center, PO Box 12692, Sana'a, Yemen

E-mail contact: dralkamel@hotmail.com
Leishmania species, genetic constitution (eg, lipophosphoglycan variation, the major abundant surface glycoconjugate, and differences in $63-\mathrm{kDa}$ glycoprotein, the major surface protease, commonly known as gp63), and the general health of the infected individual (eg, poor nutrition, advanced age). ${ }^{4,5}$

Leishmania resides as amastigotes and replicate inside the host's phagocytic cells (macrophages, dendritic cells, and neutrophils). To maintain a chronic infectious state within its host, Leishmania parasites have developed some successful strategies to prevent activation of effective immune responses and maintain the antimicrobial activities of host cells. These include remodeling the host cells' phagosomal compartments, interfering with the intracellular signaling pathways, alteration of toll-like receptor pathways, modulation of cytokines and chemokines, modification of T-cell responses, evasion of the host's cellular immunity, and generation of nitric oxide and oxygen oxidative radicals. ${ }^{6,7}$ In contrast, carcinogenesis can be influenced by several physiologic, exogenous, environmental, genotypic, and phenotypic factors. 8,9

Leishmaniasis and malignancy are both common health hazards, and some causal associations between these 2 clinical entities have been evidenced in experimental animals and in humans. However, the pathophysiologic implications of Leishmania as a carcinogenic mediator has been less well elucidated. $4,8,10$

The present report has reviewed the up-to-date data supporting the theory of the oncogenic potential of Leishmania and provided a perspective point from the Regional Leishmaniasis Control Center 
(RLCC) in Yemen, which treats an average of 100 to 150 new patients with leishmaniasis each month.

\section{Impetus and Method}

My clinical encounters at the outpatient clinics of RLCC in Yemen of several presentations of malignancy in patients with a history of leishmaniasis gave me the impetus to seek further data in support of the concept of Leishmania as a carcinogen. ${ }^{11,12}$ These malignancies included basal cell carcinoma (BCC), squamous cell carcinoma (SCC), and lymphoma (Figures 1-3). These cancers had developed in patients aged 50 to 100 years who had a history of popular treatment abuse and chronic sun exposure. These patients also had CL lesions on their face, providing evidence of Leishmania infection.

English-language studies were investigated for reports of the concurrent development of leishmaniasis disease and malignancy. However, the focus was on reported cases of the direct involvement of Leishmania spp. in the pathogenesis of malignant lesions.

\section{Associations Between Leishmaniasis and Malignancy}

The association between viral, bacteria, and parasitic infections with the development of human malignancy has been recognized for years. ${ }^{9}$ Although some parasitic infections have been very strongly associated with specific cancers, the available reported data have potentially linked leishmaniasis infection with the development of malignancy in humans and in animals. ${ }^{13,14}$ The potential associations between leishmaniasis and malignancy included the following:
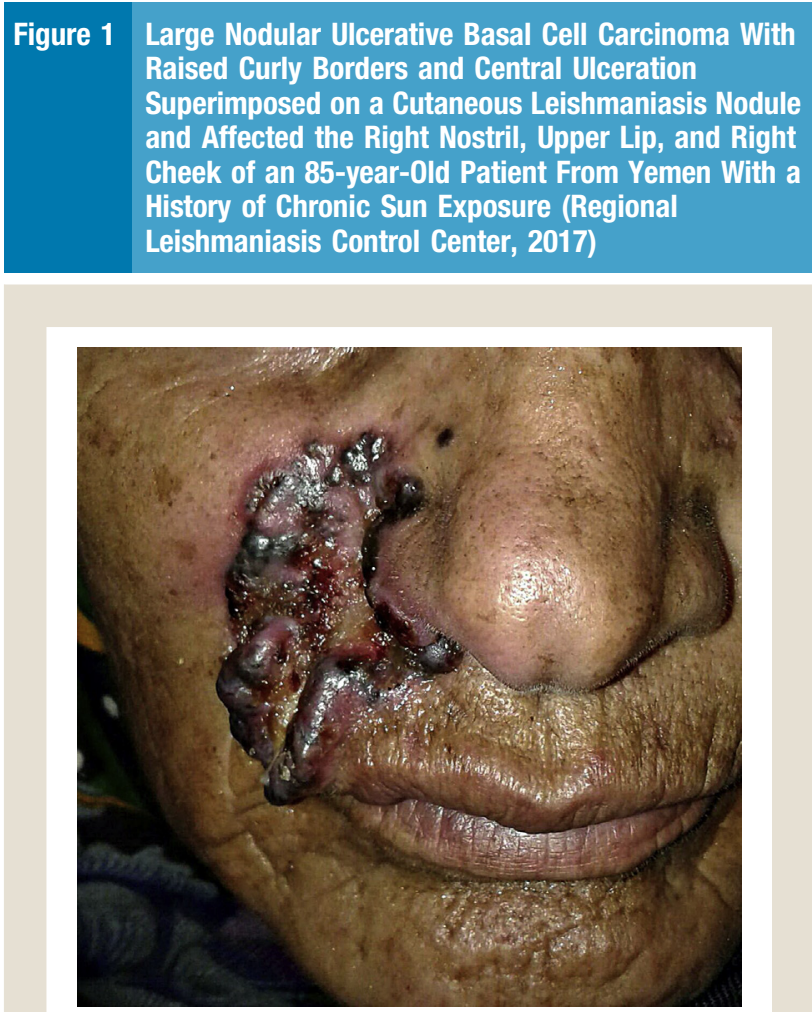

Figure 2 (A and B) Recurrent, After Surgical Excision, Basal Cell Carcinoma Ulcers That Had Developed on an Active Mucocutaneous Leishmaniasis Lesion and Destroyed the Nose of a 79-year-Old Man From Yemen (Regional Leishmaniasis Control Center, 2017)

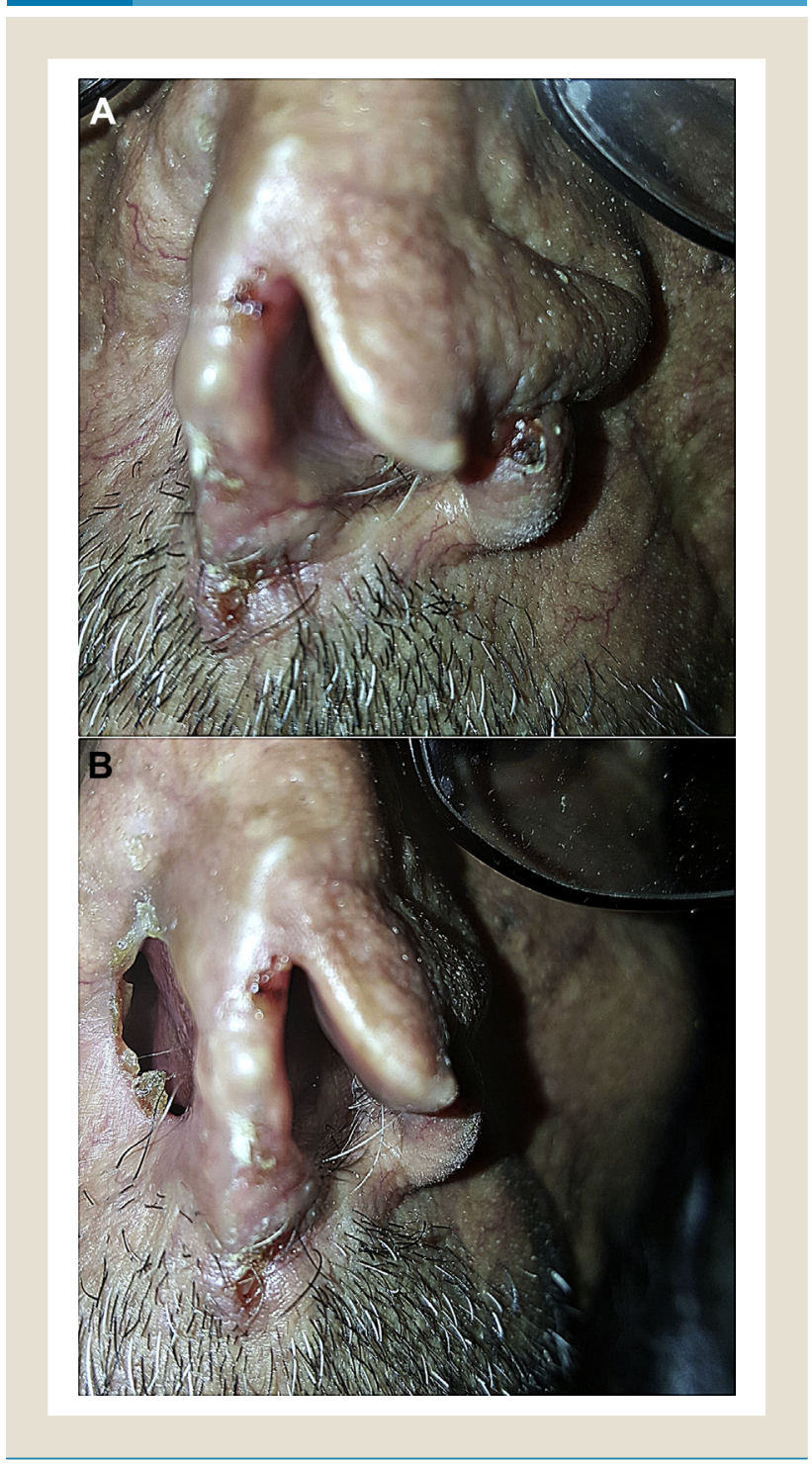

Reproduced, With permission, From Al-Kamel ${ }^{12}$

(1) leishmaniasis mimicking malignancy (Figure 4); (2) leishmaniasis coexisting with malignancy; (3) malignancy developing in patients with leishmaniasis (Figures 1-3); and (4) leishmaniasis developing in patients with malignancy. ${ }^{13}$

Numerous studies have reported a well-established relationship between Leishmania spp. and the pathogenesis of malignant lesions, including BCC, SCC, lymphoma, leukemia, and hemangiosarcoma. Amastigotes have been identified in infiltrating macrophages and within the cytoplasm of neoplastic cells from tumors with different histogenesis variants. ${ }^{15-19}$

Other illustrative reports have described the presence of large numbers of atypical mitotic features (marked dysplasia) and diffuse 


\section{Leishmaniasis and Malignancy}

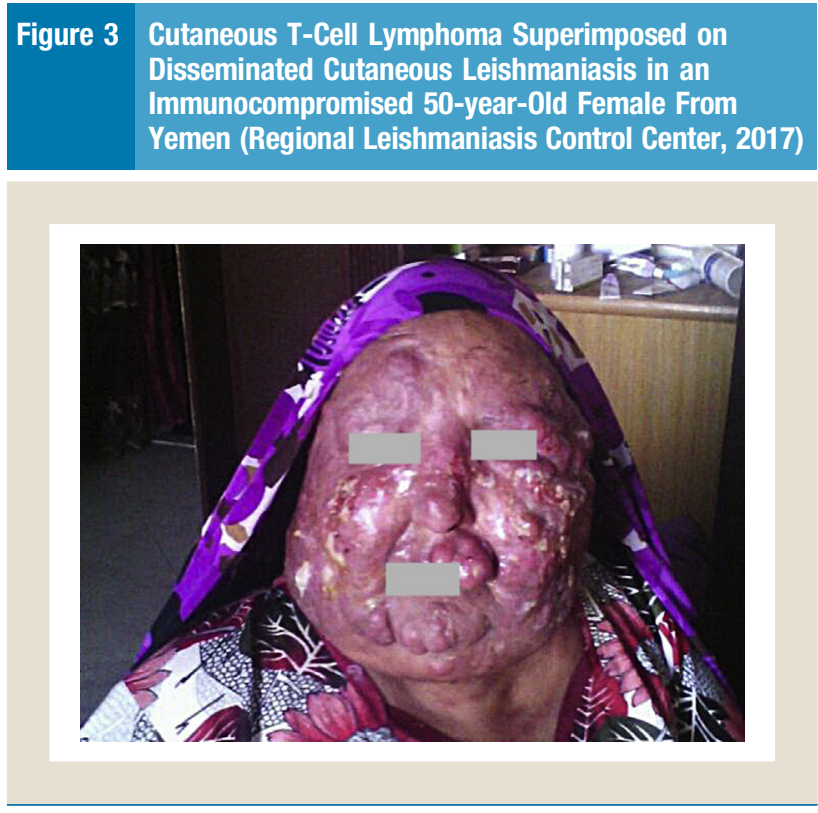

Reproduced, With permission, From Al-Kamel ${ }^{11}$

lymphoid infiltrate of predominantly B-lymphocytes, surrounded by a population of $\mathrm{CD}^{+}$and $\mathrm{CD}^{+}{ }^{+}$-lymphocytes in the excisional biopsies from patients with $\mathrm{CL}$ lesions. ${ }^{13}$ In addition, prolonged antigenic stimulation and chronic immunosuppression, typical of leishmaniasis, might play a crucial role in the etiopathogenesis of certain cancers, such as T-cell lymphoma. ${ }^{15,20}$

In 2014, a study conducted at the Research Center of the Instituto Nacional de Câncer (Rio de Janeiro, Brazil), concluded that in the differential diagnosis of childhood leukemia, VL should be considered as a potential concurrent disease in regions in which
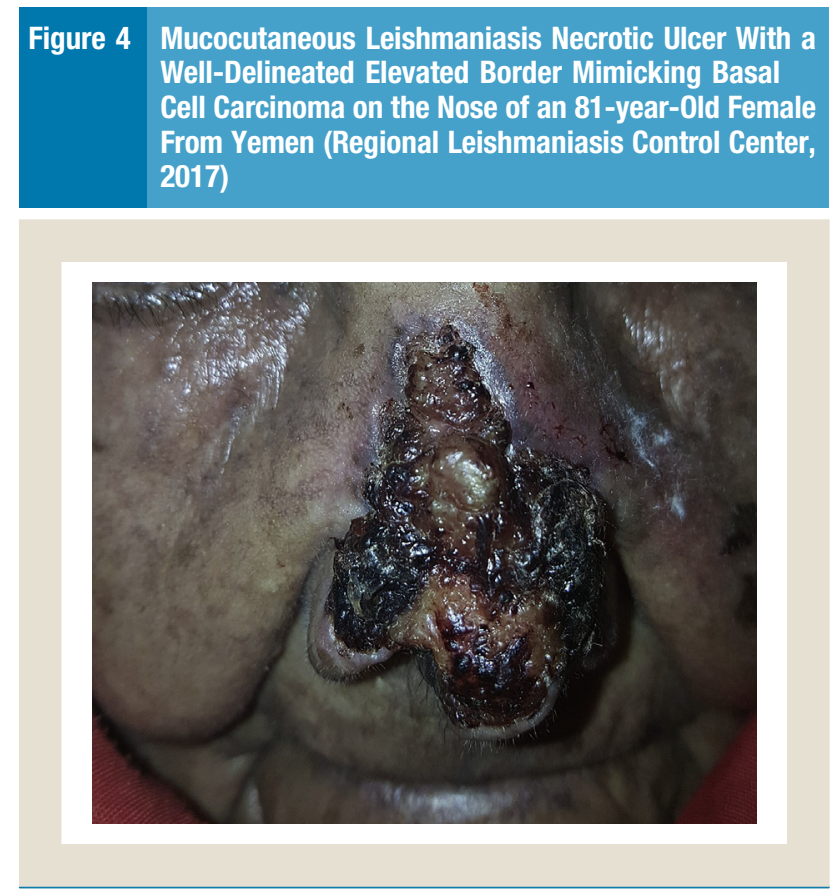

Leishmania chagasi is endemic. ${ }^{21}$ In 2013 , another study conducted in Egypt confirmed that CL allows for mutation and the development of skin cancer. ${ }^{22}$ In 2013, Nwamaka et al ${ }^{23}$ recommended that clinicians should not discount the profound immune dysregulation associated with widely disseminated infection, such as VL, and should be vigilant in including lymphoproliferative disorders and lymphomas as complications of Leishmania infection. In 2011, Kumar et $\mathrm{al}^{24}$ highlighted the mutual immunomodulation that resulted in the presentation of leishmaniasis as an opportunistic infection in a patient with Hodgkin lymphoma before chemotherapy. In 2007, Kopterides et $\mathrm{al}^{13}$ reported that a leishmaniasis-related scar is a recognized factor that predisposes to the development of malignancy. In addition, $88.9 \%$ of the studies that speculated on a possible causal association between leishmaniasis infection and the subsequent appearance of skin or mucous membrane malignancy reported that BCC, SCC, or hepatocellular carcinoma had developed at the site of healed leishmanial scars or within a few months after the first symptoms of VL, with $10.1 \%$ developing concurrently at the site of infection. ${ }^{13}$ Also, in 2005, Mangoud et $\mathrm{al}^{25}$ found dysplasia in the cells surrounding the leishmanial ulcer and concluded that leishmaniasis disease adversely affects the activation and function of macrophages and dendritic cells, which allows the transformed malignant cells to escape from immune detection and destruction. Finally, in 2005, Ozbilge et $\mathrm{al}^{26}$ reported that patients with cutaneous leishmaniasis are affected by oxidative stress, which could contribute to the progression of the disease.

Because all the patients who have presented to RLCC in Yemen with malignant disorders in addition to leishmaniasis infection were older individuals with a history of chronic use of unsafe popular treatments and sun exposure, I believe internal factors, such as aging, and external factors, such as ultraviolet radiation and abuse of popular treatments, such as animal (eg, chameleon) saliva, herbal (eg, cactus) recipes, corrosive chemicals, and topical steroids, can play a significant role in triggering a carcinogenic process (Figure 5).

\section{Pathophysiologic Cascades of Leishmaniasis-induced Malignancy}

Although the exact etiology of malignant lesions in organs infected with Leishmania parasites is still not well understood and requires further investigation, mutation research has supported the notion that leishmaniasis infection could influence carcinogenesis through numerous pathophysiologic cascades. These include chronic infection, chronic inflammation, metabolic oxidative stress, apoptosis inhibition, inhibition of tumor suppressors, wound healing, genetic transfer, nonspecific tropism, Leishmania-driven lymphoproliferation, and leishmaniasis-related scarring.

\section{Chronic Infection}

The chronic irritation hypothesis remains a widely supported mechanism for carcinogenesis by infectious agents. This is because many cancers arise from sites of infection. ${ }^{8}$

\section{Chronic Inflammation}

Recent data have expanded the concept that chronic inflammation is a critical component of the neoplastic process, fostering 

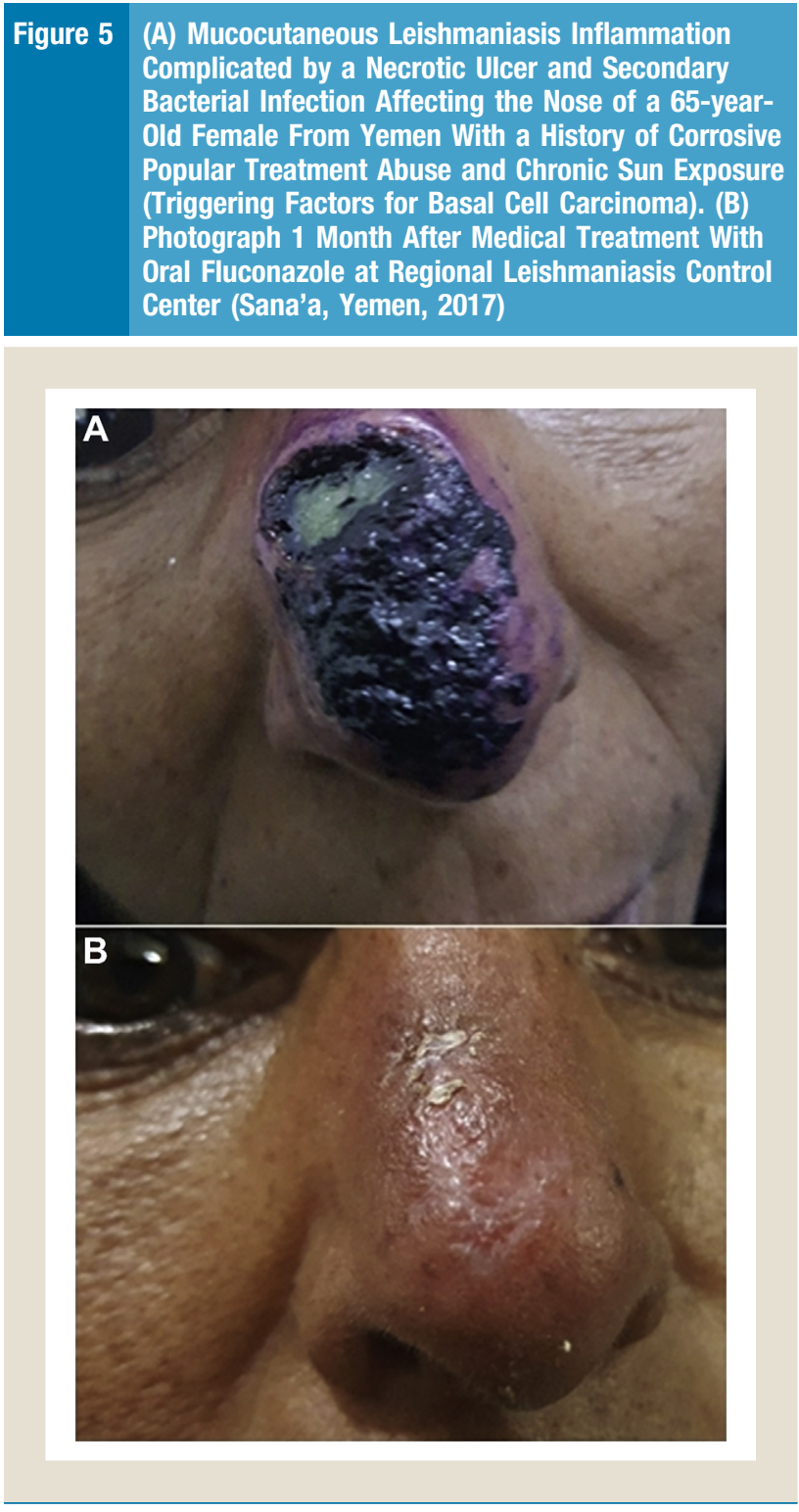

proliferation, survival, and migration of cancer cells. The chronic inflammation process due to prolonged persistence of Leishmania spp. in the host leads to repeated cycles of cell damage (caused by the host's immune response to the infection rather than by the infecting organism itself) and compensatory cell proliferation, thus orchestrating a tumor microenvironment. ${ }^{\text {? }}$

\section{Metabolic Oxidative Stress}

The reactive oxygen species and reactive nitrogen species produced by phagocytes during the inflammatory response to Leishmania infection, along with reduced total antioxidant capacity, are an important part of the host-defense strategies of organisms to kill the parasite. However, although these reactive oxidant mediators are manufactured for the purpose of killing invading Leishmania parasites, they also inflict damage on nearby tissues and induce oxidative DNA damage in noninfected cells. These have been proposed to be of a pathogenic significance and might contribute to increased mutation rates, genome instability, and cell proliferation. ${ }^{10,27}$

\section{Apoptosis Inbibition}

Many intracellular protists, such as Leishmania spp., are known to induce apoptosis inhibition, an effect that could be a significant step in the progression to malignancy. ${ }^{28}$

\section{Inbibition of Tumor Suppressors}

Leishmania spp. induce modulation of the host immune response, which could lead to inhibition of tumor suppressor surveillance mechanisms. ${ }^{29}$

\section{Wound Healing}

Host tissue damage during parasite development, along with the active wound healing, has been recognized as a carcinogenic mechanism. ${ }^{30}$

\section{Genetic Transfer}

The presence of Leishmania amastigotes has been documented in the cytoplasm of neoplastic cells from tumors of different histogenesis. The transfer of genetic material from the Leishmania parasite to the host can cause DNA damage and directly interfere with cell transformation. ${ }^{31}$

\section{Nonspecific Tropism}

The reported data have indicated the ability of Leishmania spp. to parasitize nonleukocytic neoplastic cells. The presence of Leishmania amastigotes within different types of cells indicates a nonspecific tropism for Leishmania. ${ }^{29,32}$

\section{Leishmania-driven Lymphoproliferation}

Prolonged immunosuppression and chronic leishmanial antigenic stimulation have been shown to play a crucial role in the etiopathogenesis of T-cell lymphoma. ${ }^{33}$

\section{Leishmaniasis-related Scarring}

The development of malignant lesions, in particular, BCC and SCC, at the sites of previous dermal scars is a well-recognized phenomenon. Almost all such cases develop in exposed areas. Thus, it has been hypothesized that the accompanying atrophy and decreased vascularity and adnexal structures in areas of scarring might render the affected tissues more susceptible to the effects of environmental factors, such as ultraviolet radiation. ${ }^{18,20}$

\section{Conclusion}

Leishmania spp. infection can play a significant, direct or indirect, role in the pathogenesis and prognosis of some malignant disorders. From my perspective, older age, ultraviolet radiation exposure, and popular treatment abuse, such as animal (eg, chameleon) saliva, herbal (eg, cactus) recipes, corrosive chemicals, and topical steroids, has been supposed as principal triggering factors for the development of cancer at sites of infection with Leishmania parasites. Malignancy should be considered in the differential diagnosis of leishmaniasis in endemic regions, such as 


\section{Leishmaniasis and Malignancy}

Yemen. Understanding this relationship could enrich the provision for the early diagnosis, proper management, and prompt control of cancer.

\section{Clinical Practice Points}

- The risk of leishmaniasis manifesting as a more severe entity is dependent on the infecting parasites' immune evasion potential.

- The potential associations between leishmaniasis and malignancy include leishmaniasis mimicking malignancy; leishmaniasis coexisting with malignancy; malignancy developing in patients with leishmaniasis; and leishmaniasis developing in patients with malignancy.

- Leishmania spp. infection can play a significant, direct or indirect, role in the pathogenesis and prognosis of some malignant disorders.

- Mutation research has supported the notion that leishmaniasis infection could influence carcinogenesis through numerous pathophysiologic cascades, including genetic transfer and nonspecific tropism.

- Older patient age, ultraviolet radiation exposure, and the abuse of popular treatments should be considered as principal triggering factors for the development of cancer at sites of infection with Leishmania parasites.

\section{Disclosure}

The author declares that he has no competing interests.

\section{References}

1. Ejov M, Dagne D. Strategic framework for leishmaniasis control in the WHO European Region 2014-2020 (2014), World Health Organization: Regional Office for Europe. Available at: http://www.euro.who.int/en/publications/abstracts/strategicframework-for-leishmaniasis-control-in-the-who-european-region-20142020-2014. Accessed: September 23, 2017.

2. Pace F. Leishmaniasis. J Infect 2014; 69:S10-8.

3. Al-Kamel MA. Leishmaniasis in Yemen: a clinicoepidemiological study of leishmaniasis in central Yemen. Int I Dermatol 2016; 55:849-55.

4. Desjeux P. Leishmaniasis: current situation and new perspectives. Comp Immunol Microbiol Infect Dis 2014; 27:305-18.

5. Bogdan C, Gessner A, Solbach W, Rollinghoff M. Invasion, control and persistence of Leishmania parasites. Curr Opin Immunol 1996; 8:517-25.

6. Olivier M, Gregory DJ, Forget G. Subversion mechanisms by which Leishmania parasites can escape the host immune response: a signaling point of view. Clin Microbiol Rev 2005; 18:293-305.
7. Gupta G, Oghumu S, Satoskar AR. Mechanisms of immune evasion in leishmaniasis. Adv Appl Microbiol 2013; 82:155-84.

8. van Tong H, Brindley PJ, Meyer CG, Velavan TP. Parasite infection, carcinogenesis and human malignancy. EBioMedicine 2017; 15:12-23.

9. Nilforooshan Z, Asilian A, Nilforoosh Zadeh MA. Skin carcinoma arising in Leishmania scar: report of four cases. Iran J Dermatol 2004; 8(suppl 1):11-6.

10. Benamrouz S, Conseil V, Creusy C, et al. Parasites and malignancies, a review, with emphasis on digestive cancer induced by Cryptosporidium parvum (Alveolata: Apicomplexa). Parasite 2012; 19:101-15.

11. Al-Kamel MA. Cutaneous T cell lymphoma (CTCL) superimposed on disseminated cutaneous leishmaniasis (DCL) in an immunocompromised female from Yemen. Int I Clin Dermatol Res 2017; S3:4-8.

12. Al-Kamel MA. Basal cell carcinoma developed on an active lesion of mucocutaneous leishmaniasis: a case report from Yemen. Arch Clin Dermatol 2017.

13. Kopterides P, Mourtzoukou EG, Skopelitis E, Tsavaris N, Falagas ME. Aspects of the association between leishmaniasis and malignant disorders. Trans $R$ Soc Trop Med Hyg 2007; 101:1181-9.

14. Ferro S, Palmieri C, Cavicchioli L, et al. Leishmania amastigotes in neoplastic cells of 3 nonhistiocytic canine tumors. Vet Pathol 2013; 50:749-52.

15. Foglia Manzillo V, Pagano A, Guglielmino R. Extranodal gammadelta-T-cell lymphoma in a dog with leishmaniasis. Vet Clin Pathol 2008; 37:298-301.

16. Bielory BP, Lari HB, Mirani N. Conjunctival squamous cell carcinoma harboring Leishmania amastigotes in a human immunodeficiency virus-positive patient. Arch Ophthalmol 2011; 129:1230-1.

17. Nadeem M. Mantle cell lymphoma with leishmaniasis: a rare association. J Rawalpindi Med Coll 2013; 17:150-2.

18. Unlü RE, Altun S, Ssensöz O. Leishmania scar: a risk factor for the development of basal cell carcinomas. J Craniofac Surg 2007; 18:708-10.

19. Friedman R, Hanson S, Goldberg LH. Squamous cell carcinoma arising in a Leishmania scar. Dermatol Surg 2003; 29:1148-9.

20. Chisti M, Almasri R, Hamadah I. Is cutaneous leishmaniasis a risk factor for basal cell carcinoma? Gulf J Oncol 2016; 1:64-6.

21. de Vasconcelos GM, Azevedo-Silva F, Dos Santos Thuler LC, et al. The concurrent occurrence of Leishmania chagasi infection and childhood acute leukemia in Brazil. Rev Bras Hematol Hemoter 2014; 36:356-62.

22. Morsy TA. Cutaneous leishmaniasis predisposing to human skin cancer: forty years local and regional studies. J Egypt Soc Parasitol 2013; 43:629-48.

23. Osakwe NM, Paulus A, Haggerty PF. Visceral leishmaniasis with associated immune dysregulation leading to lymphoma. Mil Med 2013; 178:e386-9.

24. Kumar R, Daga MK, Kamble NL. Rare association of visceral leishmaniasis with Hodgkin's disease: a case report. Infect Agent Cancer 2011; 6:17.

25. Mangoud AM, Sanad EM, Fouad MA. Proliferative changes of epidermal cells in lesions of cutaneous leishmaniasis. J Egypt Soc Parasitol 2005; 35:761-72.

26. Ozbilge H, Aksoy N, Kilic E, Saraymen R, Yazar S, Vural H. Evaluation of oxidative stress in cutaneous leishmaniasis. J Dermatol 2005; 32:7-11.

27. Kocyigit A, Keles H, Selek S, et al. Increased DNA damage and oxidative stress in patients with cutaneous leishmaniasis. Mut Res 2005; 585:71-8.

28. Babior BM. Phagocytes and oxidative stress. Am J Med 2000; 109:33-44.

29. Sawa T, Ohshima H. Nitrative DNA damage in inflammation and its possible role in carcinogenesis. Nitric Oxide 2006; 14:91-100.

30. Coussens LM, Werb Z. Inflammation and cancer. Nature 2002; 420:860-7.

31. Khurana S, Dubey ML, Malla N. Association of parasitic infections and cancers. Indian J Med Microbiol 2005; 23:74-9.

32. Oliveira FJ, Cecchini R. Oxidative stress of liver in hamsters infected with Leishmania (L.) chagasi. J Parasitol 2000; 86:1067-72.

33. Almeidaa BFM, Narcisoa LG, Meloa LM, et al. Leishmaniasis causes oxidative stress and alteration of oxidative metabolism and viability of neutrophils in dogs. Vet J 2013; 198:599-605. 